\title{
AC 2008-2234: A NOVEL METHODOLOGY FOR ENGINEERING COURSE DESIGN BASED ON SIX SIGMA PRINCIPLES: INCORPORATION OF DIVERSE CONSTITUENTS IN COURSE DESIGN
}

\section{Anoop Desai, Georgia Southern University}

Dr. Anoop Desai received his BS degree in Production Engineering from the University of Bombay in 1999, and MS and Ph.D. degrees in Industrial Engineering from The University of Cincinnati in 2002 and 2006. His main research interests are in Product Lifecycle Management, Design for the Environment, Total Quality Management including tools for Six Sigma and Ergonomics.

Jean-Claude Thomassian, State University of New York, Maritime College

Dr. Jean-Claude Thomassian received his BS degrees in Electrical Engineering and Mechanical Engineering from the University of Toledo in 1992 and 1993, respectively, and MS and Ph.D. degrees in Electrical Engineering from The University of Toledo in 1995 and 2002. His main professional interests are in mixed mode IC design and electrical engineering education; his recent research activity concentrates on symbolic analysis of circuits and MOS models. 


\title{
A Novel methodology for engineering course design based on Six Sigma principles: Incorporation of diverse constituents in course design
}

\begin{abstract}
This paper seeks to present a systematic and thorough methodology to incorporate basic six sigma principles for quality into engineering course design from the ground up. Six sigma principles have been widely used in industry in conjunction with the basic philosophy of 'lean thinking' so as to achieve the twin goals of quality enhancement and cost minimization. The authors intend to extend an identical thought process to the field of education, beginning at the basic course level in an engineering setting. The principal advantage of this extension is to incorporate voices of widely varying stakeholders including the community, industry, academicians etc into the basic course structure. Another advantage of implementing this thought process includes rigorous control of instruction quality across a wide spectrum of instructors thereby infusing much needed objectivity into basic course design. Different Six Sigma tools such as Quality Function Deployment (QFD), Pareto Analysis etc have been used as appropriate and a pilot study is presented at the end in order to illustrate practical utility of the methodology.
\end{abstract}

\section{Introduction}

The Six Sigma concept of product design and management has been widely utilized in commercial enterprises for over two decades now. The basic idea underlying this tenet is primarily of 'waste minimization' as well as of ensuring uniform levels of quality in terms of product characteristics as well as service characteristics (1, 2, 3, and 4). The concept of 'Total Quality Management (TQM)' is often used synonymously and sometimes in conjunction with the basic principles of lean engineering and Six Sigma.

It should be noted that while the principles of Six Sigma are generally used in the context of product design and manufacturing industries, their scope is not merely limited to these two fields of endeavor. Six sigma principles find much wider scope of expression in other industries as well. For instance, the service industry such as banking, finance, health services, restaurant management etc routinely utilize six sigma concepts in order to ensure the availability of quality products so as to gain and maintain an edge over their competitors $(5,6)$.

While the aforementioned facts are largely understood as well as followed, the authors wish to point out the fact that there has been very little formally documented research connecting a formal process of building quality to the field of education. Undergraduate as well as graduate education is conducted in institutions that are primarily 'not for profit' in terms of their formal tax status. However, as far as quality of the final product is concerned (quality of education imparted to students, in this context), it should make no difference whether the institution rendering the service is a 'for profit' institution or otherwise.

This paper seeks to extend the practically realizable as well as demonstrable advantages of the 'six sigma method of reasoning' to the field of course design. This is meant to be a starting point in a wider research attempt to finally try and extend this concept to program design. 
Quality Function Deployment (QFD) is the most basic tool used in this endeavor. It forms the crux of the design methodology and the entire thought process essentially revolves around this basic tool. However, QFD by and itself is insufficient to ensure ongoing product quality. The product (education imparted in a specific course) needs to continuously evolve by keeping pace with a variety of outside factors in the environment in terms of higher levels of knowledge, increasing as well as radically different expectations from employers, progressively higher quality of students, evolving technology etc. This factor of continuous evolution is extremely important in the context of this methodology being applied specifically to engineering course design for the sake of this paper. The general field of engineering is highly applied in nature and as such graduates of quality engineering problems tend to exhibit two principal characteristics:

- Be readily employable under sound economic conditions

- Be able to contribute productively to the existing knowledge base in order to enhance and further the theory as well as practice of engineering.

It needs to be noted that continuous evolution is somewhat different than the rather well known concept of continuous improvement. While any process can be continuously improved within the same set of parameters by means of input optimization, continuous evolution, in essence seeks to capture changing trends in input parameters themselves. The process of continuous improvement can then be recursively applied to the evolved product.

This paper is divided into two parts. The first part seeks to briefly present the methodology. The second part seeks to demonstrate practical utilization of this methodology to real life course design.

\section{Methodology}

The design methodology is comprised of three main components as listed below:

\section{- Utilizing Quality Function Deployment:}

A QFD centric approach forms the basis of course design. This approach enables the incorporation of the opinions as well as the preferences of various constituencies such as potential employers, accreditation boards, parents, state and national professional regulation boards etc. This step utilizes the four stage 'House of Quality' approach to quantify priorities.

1. Course Planning: This step incorporates the voice of end users (employers, accreditation board, professional development societies etc). This step is akin to asking the question: 'What outcome do you expect out of this course?' Once these opinions are ascertained, they are prioritized in arrange from highest priority to lowest priority

2. Component Deployment: This step breaks down the customer needs to product characteristics. For instance, a customer need such as 'hands on experience in programming' could be translated into components such as 'knowledge of programming theory', 'knowledge of programming practice', 'knowledge of debugging' etc.

3. Instruction Planning: This step identifies the resources and methods essential to be able to impart sound fundamental knowledge in keeping with customer preferences. 
4. Quality Control: This step identifies the minimum level of knowledge expected out of all students getting at least a passing grade in the course.

5. With some minor modifications, the voice of students as reflected in their evaluations can also be incorporated into this methodology. However, such a modification is outside of the scope of this paper and is not discussed here.

The House of Quality is the principal tool used in QFD. It is depicted in figure 1.

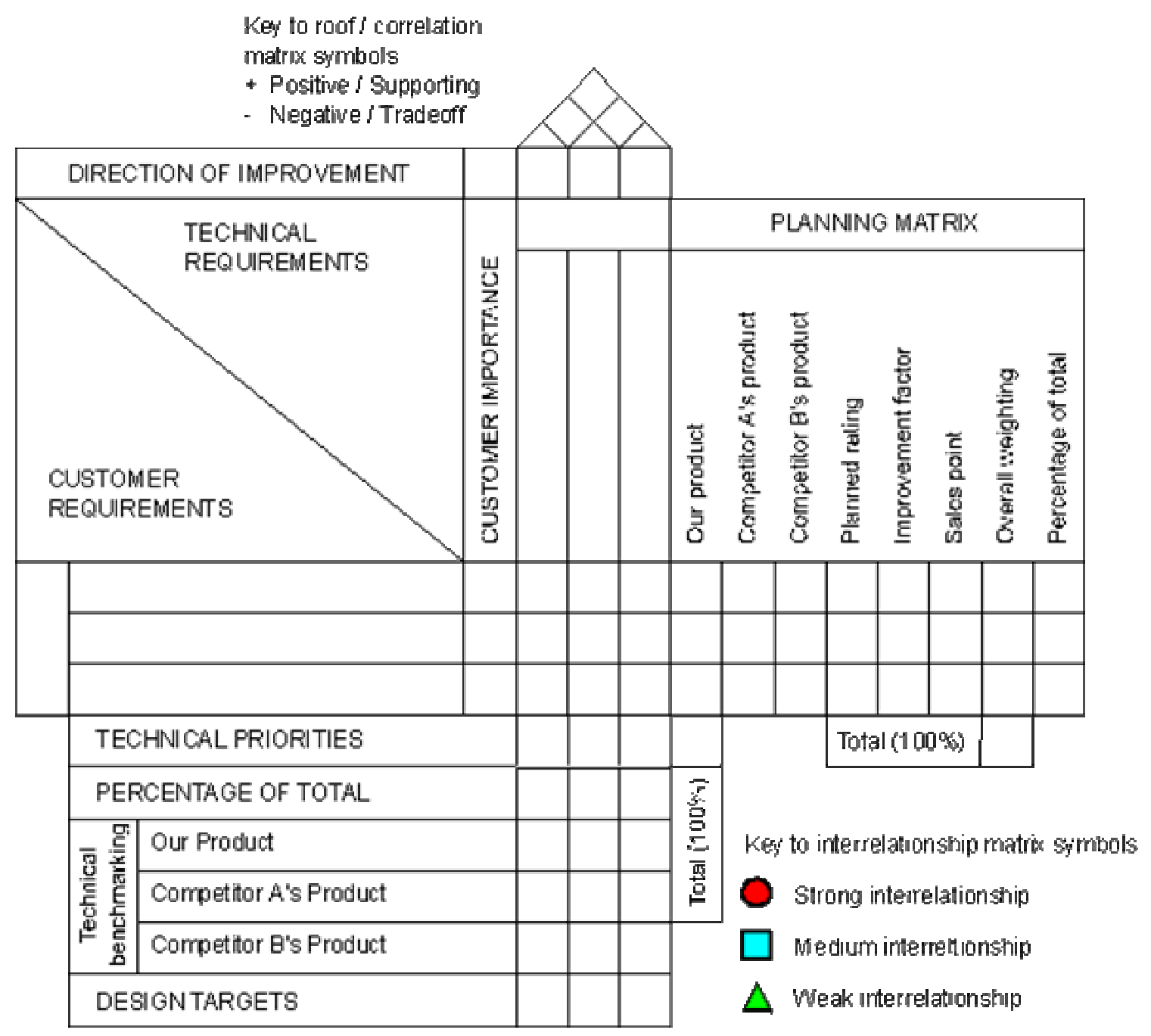

Figure 1: The House of Quality (QFD)

The customer requirements are entered in the left column and are translated into course characteristics under technical requirements. The customer requirements are prioritized under the customer importance column. An interesting feature of using this approach is to compare characteristics of the course with similar courses available at other institutions so as to be able to attract more students. Each customer requirement is matched with product characteristic 
depending on whether the relationship between them is strong, medium or weak. Non-existent relationships are not marked.

Each successive iteration of the house of quality results in different inputs under the left column and successively different inputs under the horizontal row. This process will be demonstrated in a short case study at the end of the paper.

\section{- Utilizing Ishikawa Analysis and Pareto Analysis to enable Continuous Improvement:}

After a course is designed and has been in use, a few anomalies and undesirable characteristics are bound to creep in over time. This stage of the methodology seeks to identify and eliminate these undesirable traits by using Ishikawa analysis and Pareto analyis.

Ishikawa analysis is characterized by the cause and effect relationship that underlies most non-conformity. Once the defect in instruction or student learning is addressed, Pareto Analysis can be applied so as to achieve further improvement of course content and instruction methods.

Ishikawa analysis is also referred to as cause and effect analysis or the fishbone diagram. It is illustrated in figure 2 .

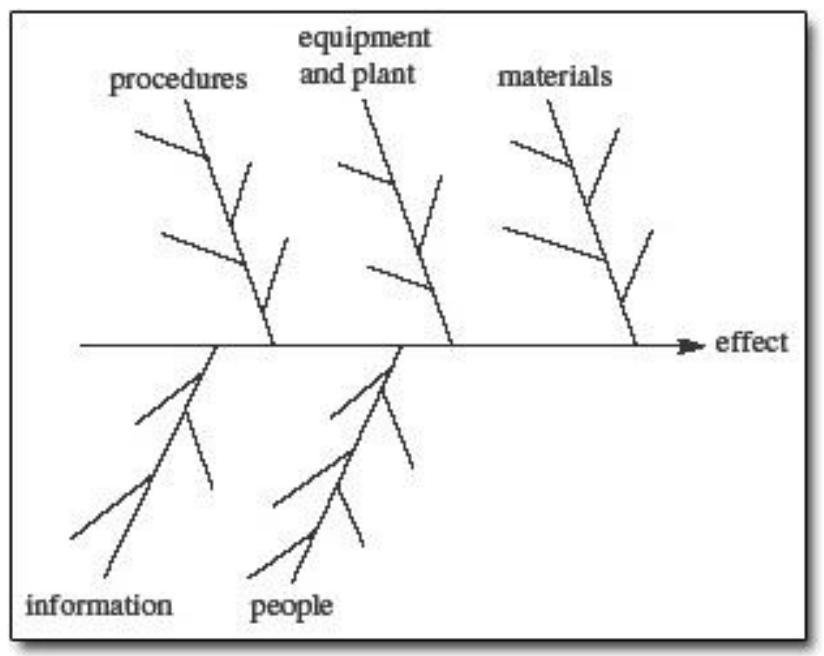

Figure 2: Example of Ishikawa Analysis for a manufacturing Environment

Pareto analysis seeks to find out the few problems that are responsible for the highest loss of student interest. This is in accordance with the general principle which in essence implies that $70 \%$ of all problems are cause by $10 \%$ of causes. Addressing the roots of these causes in conjunction with Ishikawa analysis leads to improvement in instruction.

\section{- Utilizing Pareto Analysis in order to enable Continuous Evolution:}

This approach constitutes the third stage of the methodology. This is an approach that seeks to weed out non-performing and obsolete components of a course by means of utilizing Pareto 
Analysis. Pareto analysis is a statistical technique in decision making that is used for selection of a limited number of tasks that produce significant overall effect. It uses the Pareto principle - the idea that by doing $20 \%$ of work one can generate $80 \%$ of the advantage of doing the entire job. Or in terms of quality improvement, a large majority of problems $(80 \%)$ are produced by a few key causes $(20 \%)$.

Pareto analysis is a formal technique useful where many possible courses of action are competing for attention. In essence, the problem-solver estimates the benefit delivered by each action, then selects a number of the most effective actions that deliver a total benefit reasonably close to the maximal possible one.

An example of Pareto Analysis is represented in figure 3.

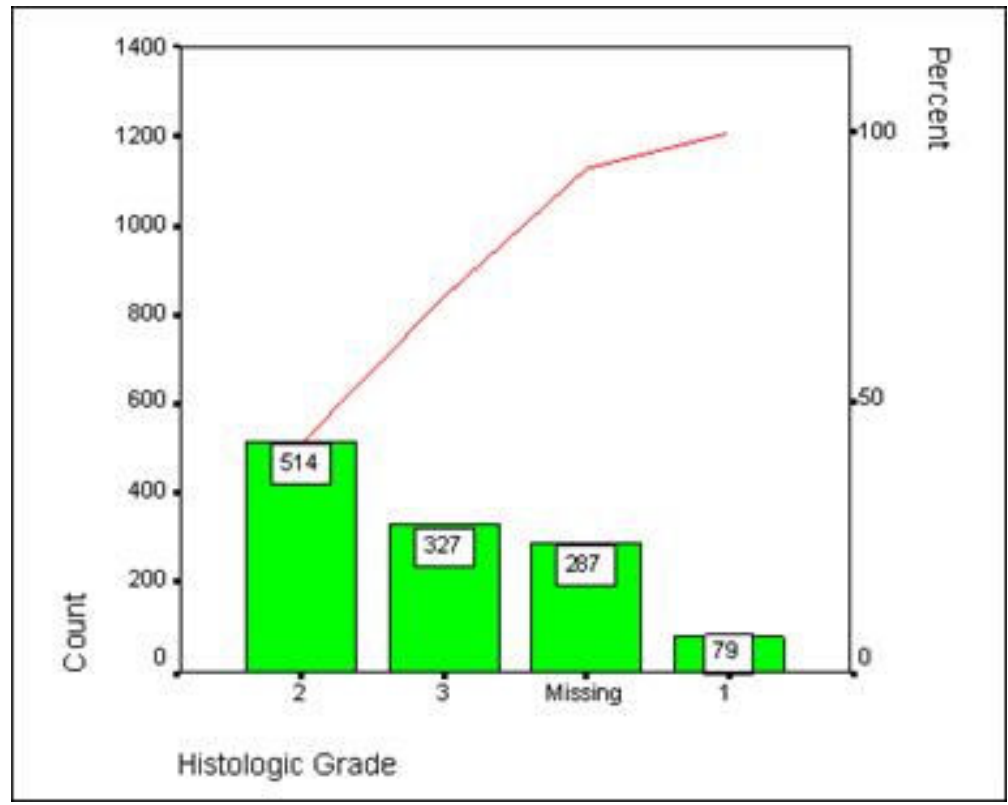

Figure 3: Example of Pareto Analysis

The design methodology is presented in figure 4. 


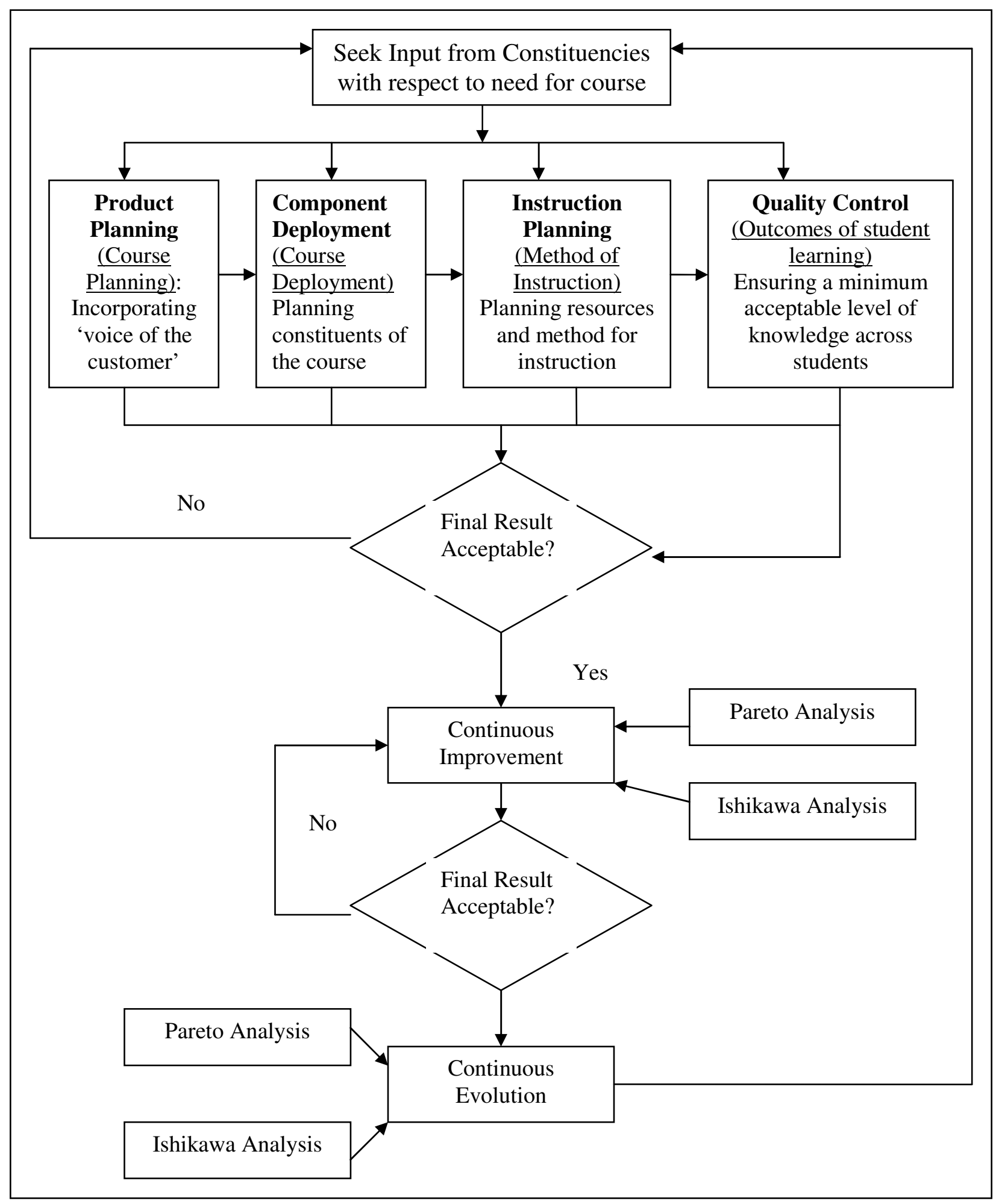

Figure 4: Methodology of using Six Sigma principles to design a course 


\section{Case Study}

\section{Stage 1: QFD Composition}

The aforementioned methodology was applied to an introductory course in Digital Computation at Georgia Southern University. This course is a substantial revision of an older course. The revision was carried out with the express purpose of addressing the needs of industry as well as academic stakeholders. Various industries in the southern Georgia area have been asking for a technical workforce that has a considerably high degree of proficiency in programming techniques owing to the widespread automation occurring in industry.

Different stakeholders including employers, professional societies, accreditation boards etc were consulted for input in terms of their expectations from students graduating from the course. It is inappropriate for every single stakeholder to be identified in this paper at this point. It is sufficient to say that stakeholders representing a wide spectrum of interests were consulted. Meetings took place over the course of a month and a half. Consensus was sometimes easy to reach and not so easy at other times. However, conflict of interest was generally resolved based on one single factor: employability. The study was done within the department and quality was monitored over a range of three instructors. The average class size was approximately 30 students each semester.

The ranking of preferences was done in direct consultation and agreement with stakeholders since for the most part they represented a majority of future employers. Questionnaires were prepared that represented both the subjective as well as objective opinion of every stakeholder. These opinions were ranked on a scale of 1 to 10 and these scores were subsequently converted into the ranking matrix. These preferences were also ranked ranging from highest importance to lowest importance. The Course planning (product planning) QFD matrix was developed in which customer preferences were converted into course (Product) characteristics. This is represented in figure 5. Successive iterations of the QFD were performed in order to convert product planning into the quality control matrix as depicted in figure 3 . The entire sequence is not being depicted here for reasons of space. Despite this, figure 5 is an excellent example of application of a QFD tool to course design.

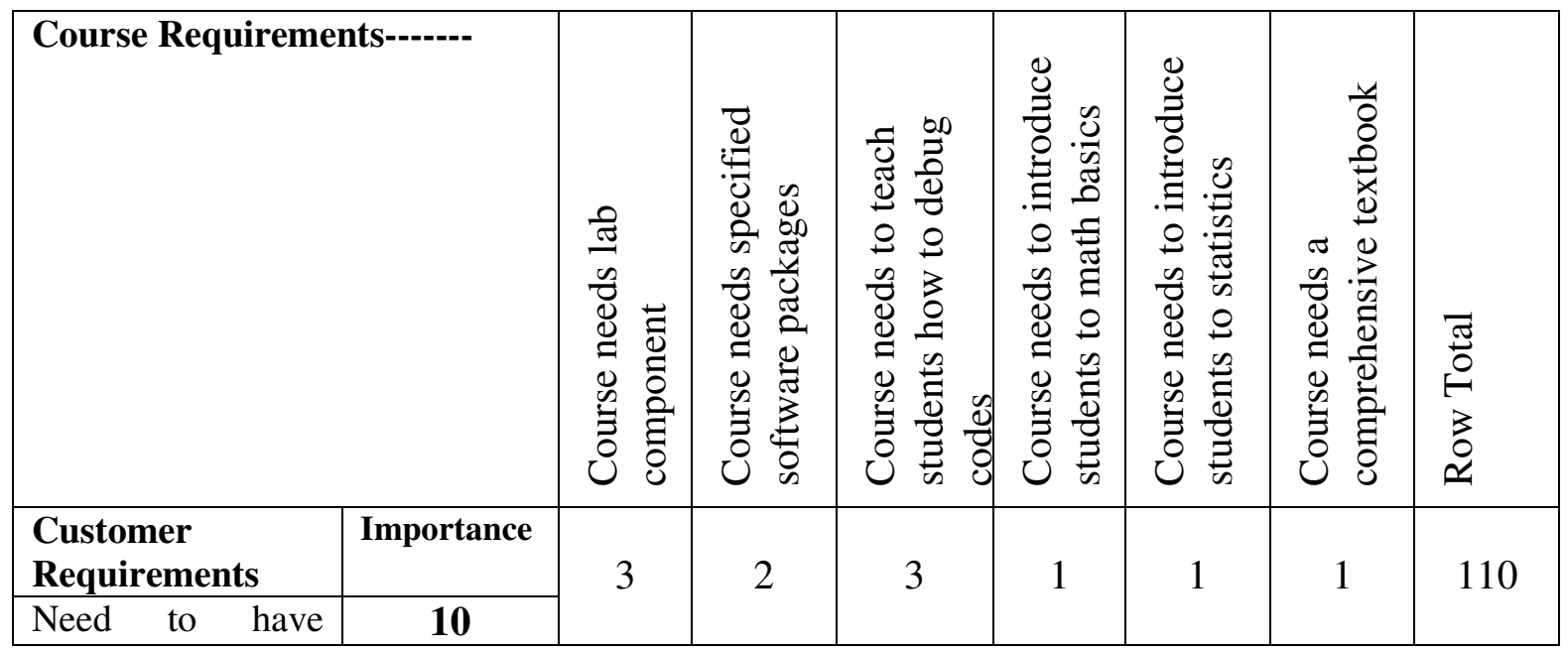




\begin{tabular}{|c|c|c|c|c|c|c|c|c|}
\hline \multicolumn{9}{|l|}{$\begin{array}{|ll|}\text { hands } & \text { on } \\
\text { experience } & \text { in } \\
\text { programming } & \\
\end{array}$} \\
\hline $\begin{array}{l}\text { Need to be } \\
\text { conversant with } \\
\text { specific programs }\end{array}$ & 8 & 2 & 3 & 3 & 2 & & 1 & 88 \\
\hline $\begin{array}{l}\text { Need to have a } \\
\text { strong quantitative } \\
\text { skill set }\end{array}$ & 7 & - & - & - & 3 & 3 & - & 42 \\
\hline $\begin{array}{l}\text { Need to be able to } \\
\text { use spreadsheets }\end{array}$ & 7 & 3 & 2 & - & - & 3 & - & 56 \\
\hline $\begin{array}{l}\text { Need to be able to } \\
\text { develop codes to } \\
\text { solve } \\
\text { problems engg } \\
\text { limited complexity }\end{array}$ & 5 & 3 & 2 & 3 & 2 & 1 & - & 60 \\
\hline Column To & & 11 & 9 & 9 & 8 & 8 & 2 & - \\
\hline
\end{tabular}

Figure 5: Example of converting customer requirements into Course requirements: First stage of the QFD iteration. Case study for an introductory course in Digital Computation

Each customer requirement is assigned a score in the 'importance' column depending on a vote of the different constituencies. The interrelationship between customer requirements and course requirements is assigned score (3, if the interrelationship is very strong, 2 is it is medium and 1 if it is weak). The row total is obtained by adding up scores of the interrelationships for each row and multiplying that number by the score assigned to importance for that row.

This iteration is done 4 times, changing the matrix each time. For instance, the second iteration matrix will constitute course requirements as rows which will be converted into constituents for the course and so on.

\section{Stage 2: Continuous Improvement and Evolution using Ishikawa analysis and Pareto Analysis}

Once the course was designed and had been taught for over 3 semesters, it was noticed that some of the original codes had become obsolete due to advances in programming technology. For instance, some of the codes wouldn't work due to changes made in Visual C++ code structure. It was very frustrating to students not to have the code work because of technical difficulties. This problem was a relatively simple one to rectify. The cause and effect were known and were put into action accordingly. Also, the sources of students' questions pertaining to programming and implementation (areas possibly needing more explanation) were identified using Pareto Analysis. It was observed that most questions could be traced back to lack of understanding of only a few basic concepts. This was done every semester that the course was delivered and for every single instructor as well.

It is essential to be pointed out that fishbone diagrams were not used to debug the VC++ code. The debugging process was carried out using normal routines. The fishbone analysis was used to identify problems and bottlenecks in course design and instructional quality. 
It has to be noted that since the time period over which the course was monitored was less than 2 years, course improvement tended to overlap with course evolution to a large extent. In the event that the course was taught over a period of over 10 years, the process of evolution would differ significantly from improvement in that new technologies as well as breakthrough achievements in that specific field would have to be adequately represented in the course material. The authors wish to point out that in order to achieve continuous improvement and evolution, the twin approaches of Ishikawa analysis and Pareto analysis need to be used in conjunction.

\section{Conclusion}

This paper demonstrated a methodology to apply basic principles of six sigma and quality improvement to the field of academics. This is an ongoing research effort and it is the authors' intention to try and publish results of their research as the process evolves over time. Nevertheless, it is very clear that the concepts of 'six sigma' and 'lean thinking' can be used very advantageously to the process of course design and can achieve substantial streamlining of the process.

\section{References}

1. Kolarik, W.J (1995), Creating Quality: Concepts, Systems, Strategies and Tools, McGraw Hill, USA

2. Akao, Y (ed) (1990), Quality Function Deployment, Cambridge, MA: Productivity Press

3. Juran, J.M (1988), Juran on planning for Quality, New York, Free Press

4. Taguchi, G (1986), Introduction to Quality Engineering: Designing quality into products and processes, White Plains, NY: Kraus International, UNIPUB (Asian Productivity organization)

5. Shingo, S (1986), Zero Quality Control: Source Control and the Pokayoke system, Cambridge, Massachusetts, Productivity Press

6. Mizuno, S (1988), Company wide total Quality Control, Tokyo: Asian Productivity Organization 